\title{
XML Data Integration using Fragment Join
}

\author{
Jian Gong, David W. Cheung, Nikos Mamoulis, and Ben Kao \\ Department of Computer Science, The University of Hong Kong \\ Pokfulam, Hong Kong, China \\ \{jgong, dcheung, nikos, kao\}@cs.hku.hk
}

\begin{abstract}
We study the problem of answering XML queries over multiple data sources under a schema-independent scenario where XML schemas and schema mappings are unavailable. We develop the fragment join operator - a general operator that merges two XML fragments based on their overlapping components. We formally define the operator and propose an efficient algorithm for implementing it. We define schema-independent query processing over multiple data sources and propose a novel framework to solve this problem. We provide theoretical analysis and experimental results that show that our approaches are both effective and efficient.
\end{abstract}

\section{Introduction}

Data integration allows global queries be answered by data that is distributed among multiple heterogeneous data sources [1]. Through a unified query interface, global distributed queries are processed as if they were done on a single integrated data source. To achieve data integration, a schema mapping is often used, which consists of a set of mapping rules that define the semantic relationship between the global schema and the local schemas (at the data sources). In these systems, such as Clio [2], processing a global query typically involves two steps: query rewriting, and data merging.

While much work has been done on query rewriting, very little has been done on data merging. In most existing approaches, data merging is mostly an ad hoc computation - a special data merging routine is custom-coded for each mapping rule. This approach leads to inflexible system design. In this paper we propose a schema independent framework that allows data merging be processed without referring to any specific schema mapping rules. and is independent of any mapping rules.

Let us illustrate our idea by an example. Figure 1(a) shows two XML documents taken from UA Cinema website and IMDB website, respectively. . Both UA and IMDB contain the title and the director of each movie. In addition, UA contains venue and price, while IMDB contains the movie's reviews. Consider a user who wants to find out the title, director, price, and review for each movie. This is expressed by the twig pattern query shown in Figure 1(b). Note that neither UA nor IMDB can answer the query alone because UA lacks reviews and IMDB lacks pricing information. The (global) query thus has to be broken into two query fragments, one for each site. The returned results from the two sites should then be merged based on their common components. Figure 1(c) shows an example of the query result. Our goal is to answer such twig pattern queries in a schema-independent fashion where mapping rules are not needed. 


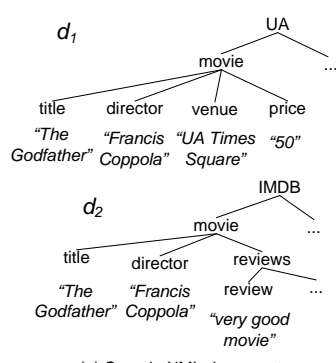

(a) Sample XML documents

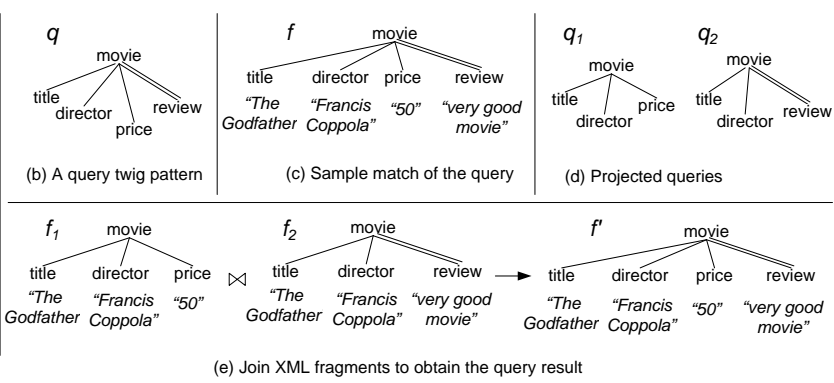

(e) Join XML fragments to obtain the query result

Fig. 1. Query on sample XML documents and the results.

In our approach, we join data fragments based on their overlapping content in order to answer queries. For example, we first project the global query on the two XML documents and obtain two local queries (Figure 1(d)). Then, we retrieve XML fragments $m(t, d, p)$ from UA and $m(t, d, r)$ from IMDB. Afterward, we join these fragments based on their overlapping parts, which are title $(t)$ and director $(d)$ (Figure 1(e)).

\section{Preliminaries}

An XML document $D$ is a rooted, node-labeled tree $D=\langle N, E, r\rangle$, wherein $N$ is a node set, $E \subseteq N \times N$ is an edge set, and $r \in N$ is the root node. Each node in an XML document has a label and may contain some text. The vocabulary of an XML document $d$, denoted by $v(d)$, is the set of distinct node labels of $d$.

Definition 1. (XML Fragment) An XML fragment $f$ is an edge-labeled XML document, where each edge is labeled by either "/" (parent-child edge) or "I//" (ancestordescendant edge). An XML fragment $f$ is a fragment of an XML document d, denoted as $f \sqsubseteq d$, if there exists an injective mapping $\lambda: f . N \rightarrow d . N$, such that: $(i) \forall n \in f . N$, $n=\lambda(n)$, and (ii) $\forall e\left(n_{1}, n_{2}\right) \in f$.E labeled as "/" (resp., "//"), $\lambda\left(n_{1}\right)$ is the parent (resp., ancestor) of $\lambda\left(n_{2}\right)$.

Definition 2. (TwIG PATTERN AND MATCH) A twig pattern is an XML fragment, where the text content of the nodes is disregarded. A fragment $f$ is a match to a twig pattern $q$, denoted as $f \vdash q$, if there exists a mapping $\gamma: q . N \rightarrow f . N$, such that the node labels and edges of $q$ are preserved in $f$. A fragment $f_{1}$ is contained in another fragment $f_{2}$, denoted as $f_{1} \preceq f_{2}$, if all the nodes and edges of $f_{1}$ are contained in $f_{2}$.

Definition 3. (PROJECTION) Given a fragment $f$ and a vocabulary $v(d)$ of a document $d$, the projection of $f$ on $v(d)$, denoted as $\rho_{v(d)}(f)$, is obtained by removing from $f$ all the nodes whose labels are not in $v(d)$ and the corresponding connecting edges.

\section{The fragment join operator}

Definition 4. (FRAGMENT JOIN) Given a set of of fragments $f_{1}, \ldots, f_{n}(n \geq 2), a$ fragment $f$ is a join of $f_{1}, \ldots, f_{n}$, denoted as $\left(f_{1}, \ldots, f_{n}\right) \stackrel{\bowtie}{\longrightarrow} f$, if $\exists f_{1}^{\prime} \preceq f, \ldots, f_{n}^{\prime} \preceq f$, 




(a) The fragment $f_{1}$ of $d_{1}, f_{2}$ of $d_{2}$

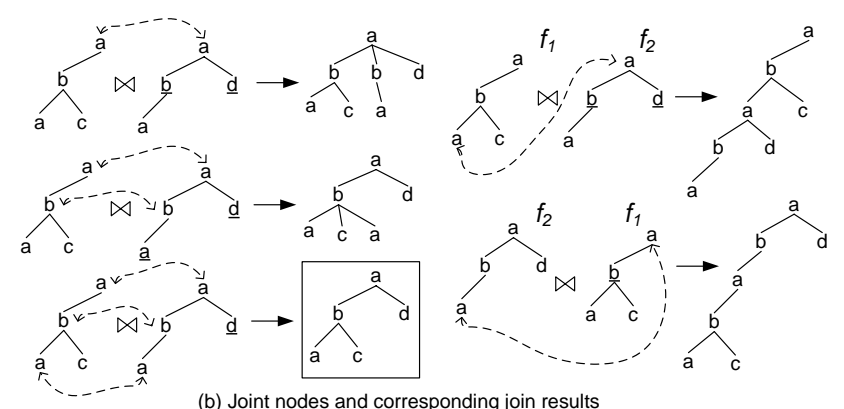

(b) Joint nodes and corresponding join results

Fig. 2. XML fragment join on different joint sub-trees.

such that: 1) $f_{i}^{\prime}=f_{i}, 1 \leq i \leq n$, 2) $\forall n \in f . N, n \in f_{1}^{\prime} . N \cup \ldots \cup f_{n}^{\prime} . N$, and 3) $\forall e \in f . E, e \in f_{1}^{\prime} . E \cup \ldots \cup f_{n}^{\prime} . E$.

In addition, the join set of $f_{1}, \ldots, f_{n}$ is a set of fragments $F=\left\{f \mid\left(f_{1}, \ldots, f_{n}\right) \stackrel{\bowtie}{\rightarrow} f\right\}$, denoted as $\left(f_{1}, \ldots, f_{n}\right) \stackrel{\bowtie}{\Rightarrow} F$.

Definition 5. (JOINT SUB-TREe) Given two fragments $f_{1}$ and $f_{2}$, a subtree $j$ s is a joint sub-tree of $f_{1}$ and $f_{2}$ if (1) js $\preceq f_{1}, j s \preceq f_{2}$, (2) the root of $j s=$ the root of $f_{2}$.

Figure 2(b) shows the five results of the fragment join between $f_{1}$ and $f_{2}$ shown in Figure 2(a). Each of these results is based on a joint sub-tree, whose nodes are pointed by double-arrowed dashed lines in the two fragments.

We propose Algorithm 1 for evaluating the fragment join of two fragments $f_{1}$ and $f_{2}$. For example, consider the first join result shown in Figure 2(b). The joint-subtree for this join result consists of a lone node $a$. The boundary nodes are the children of the root node $a$ in $f_{2}$, which are labeled $b$ and $d$ (underlined). The subtrees of these boundary nodes are attached to the matching node $a$ in $f_{1}$ forming the join result.

\section{Schema-independent, query-based data integration}

Our research problem is formally stated as following: given XML documents $d_{1}$ and $d_{2}$, and a twig pattern query $q$, compute $F=\left\{f \mid f \vdash q ;\left(f_{1}, f_{2}\right) \stackrel{\bowtie}{\longrightarrow} f ; f_{1} \sqsubseteq d_{1} ; f_{2} \sqsubseteq d_{2}\right\}$. Our approach to solve this problem consists of the following phases.

Projection. The twig query $q$ is rewritten into local queries $q_{1}=\rho_{v\left(d_{1}\right)}(q)$ and $q_{2}=\rho_{v\left(d_{2}\right)}(q)$ using the project operator (Section 2). We then apply the fragment join operator on $q_{1}$ and $q_{2}$ to find a joint sub-tree $j s$ for which the join result is $q$.

Matching. Two sets of fragments $F_{1}$ and $F_{2}$ are returned, which contains all matches to the local query $q_{1}$ in $d_{1}$ and all matches to the local query $q_{2}$ in $d_{2}$, respectively ${ }^{1}$.

Join. For each pair of fragments $\left(f_{1}, f_{2}\right) \in F_{1} \times F_{2}$, we compute the fragment join of $f_{1}$ and $f_{2}$ using the joint-subtree obtained in the projection phase. The join results are returned as the query's answer.

\footnotetext{
${ }^{1}$ We thank the authors of [3] for providing us with the implementation of TwigList, used as a module for evaluating twig queries in our work.
} 


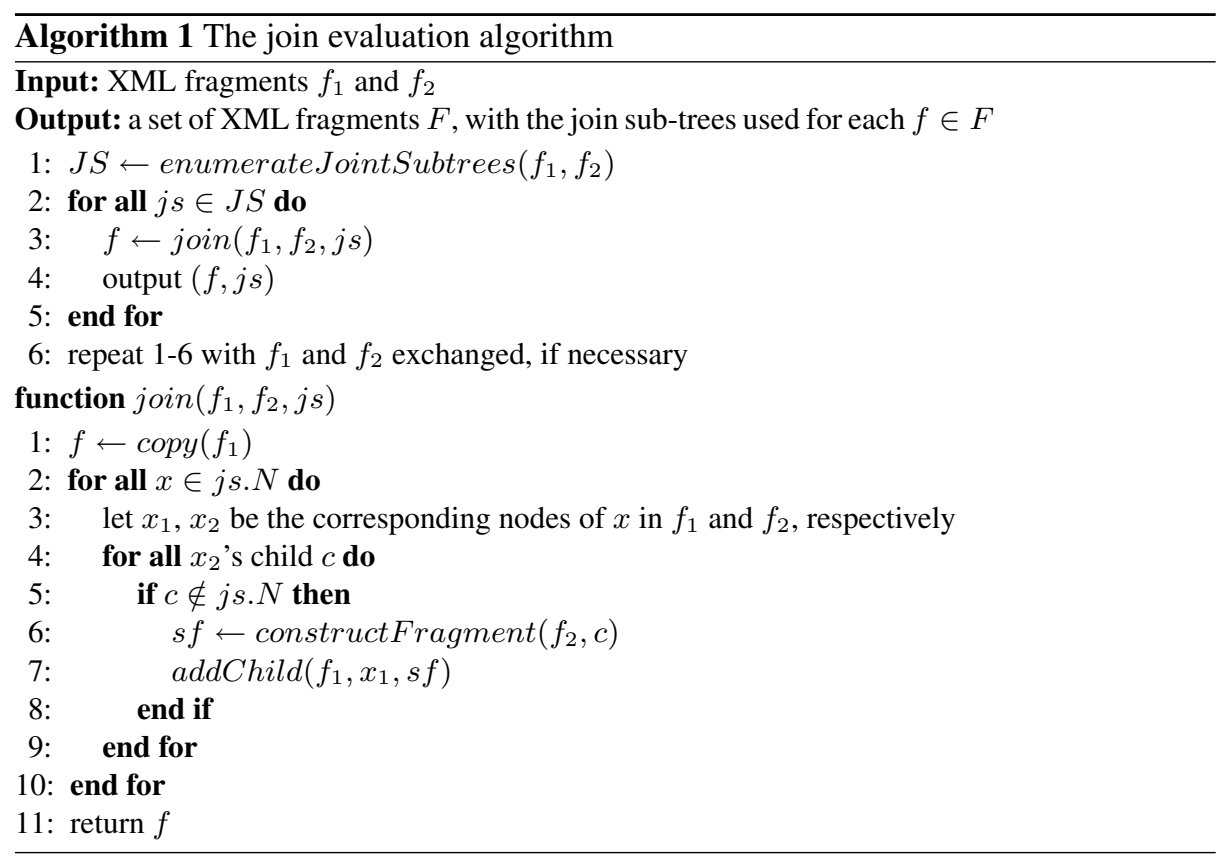

Figure 3 illustrates our approach (the found joint sub-tree contains the underlined nodes). We note that projecting a global query onto local sources so that one single local query is applied to each source may not be sufficient to retrieve the complete set of query results. For example, consider again query $q$ in Figure 3 . We observe that joining $q_{11}$, a sub-twig pattern of $q_{1}$ containing nodes $b$ and $c$ and the edge between them, with $q_{2}$ also gives us $q$ (using the joint sub-tree $b$ ). Therefore, in order to ensure that all valid query results are found, we should consider all pairs of sub-twig patterns of $q_{1}$ and $q_{2}$ that can form $q$.

Definition 6. (RECOVERABILITY) Given a twig pattern $q$, a pair of twig patterns $\left(q_{i}\right.$, $\left.q_{j}\right)$ is recoverable for $q$, denoted as $\left(q_{i}, q_{j}\right) \stackrel{r}{\sim} q$, if $\left(q_{i}, q_{j}\right) \stackrel{\bowtie}{\rightarrow} q$ using some joint sub-tree $j s$; else, $\left(q_{i}, q_{j}\right)$ is non-recoverable for $q$, denoted as $\left(q_{i}, q_{j}\right) \stackrel{r}{\chi_{i}} q$.

We add two more schema-level phases to the Projection-Matching-Join framework, in order to ensure completeness of the query results.

Decomposition. After the projection phase in which local queries $q_{1}$ and $q_{2}$ are derived, the decomposition phase returns: $Q_{1}=\left\{q_{i} \mid q_{i} \preceq q_{1}\right\}$, and $Q_{2}=\left\{q_{j} \mid q_{j} \preceq q_{2}\right\}$.

Recoverability checking. After the decomposition phase, this phase returns: $\left\{\left(q_{i}, q_{j}\right)\right.$ $\left.\mid\left(q_{i}, q_{j}\right) \in Q_{1} \times Q_{2} \wedge\left(q_{i}, q_{j}\right) \stackrel{r}{\sim} q\right\}$.

\section{Experimental evaluation and conclusion}

We use the DBLP and CiteSeer datasetsin our experiments. The raw CiteSeer data are in plain text BibTeX format. We converted them into an XML file having similar schema 


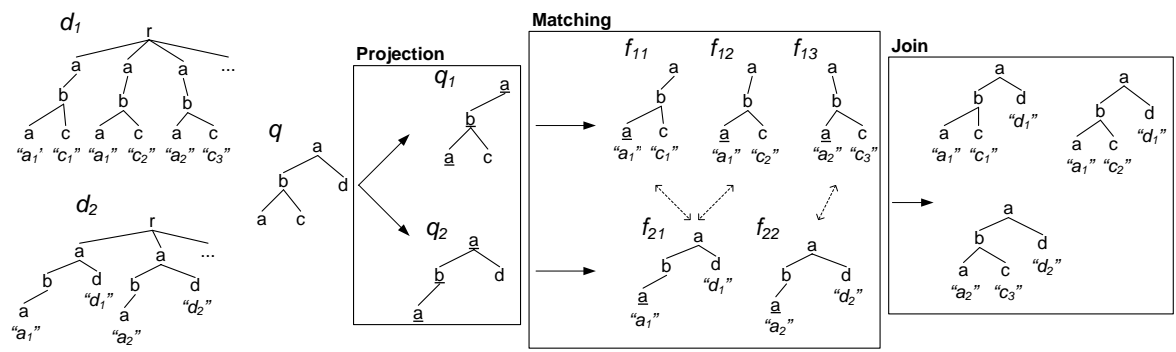

(a) Two XML documents

(b) The twig pattern query and Projection-Matching-Join query answering process

Fig. 3. Query answering from multiple data sources: projection, matching, and join.



Fig. 4. Overall performance of PDRMJ for all queries and datasets.

to that of DBLP data. The size of Citeseer dataset is $15 \mathrm{MB}$. We randomly sample the original DBLP (130MB) dataset to extract the publication records and attributes, and obtain five DBLP datasets, whose sizes are: 1MB, 10MB, 20MB, 40MB, and 80MB, respectively. Thus, we have five pairs of datasets used for the queries, each consisting of the Citeseer dataset plus one of the sampled DBLP datasets.

We manually created four test twig pattern queries, named Q1-Q4, each of which queries on a set of atrributes of papers, such as title. All these queries can only be answered using both DBLP and Citeseer datasets (but not one of the two datasets alone) by fragment join in our framework.

The overall performance of our complete, optimized approach (PDRMJ) is tested in Figure 4 for all queries Q1-Q7 on all datasets. The overall response time is broken down to two parts: (i) the time spent by all sub-twig pattern queries issued against the different sources, and (ii) the time spent by the fragment joins. We observe that the performance for all queries scales roughly linearly to the size of the DBLP dataset (recall that the size of the CiteSeer dataset is fixed). In addition, nearly half of the cost is due to the twig pattern queries against the sources.

In conclusion, we developed a fragment join operator for query-based data integration from multiple sources. We studied the problem of schema-independent data integration based on this operator. We used experiments to support the effectiveness of our approaches.

\section{References}

1. Lenzerini, M.: Data integration: a theoretical perspective. In: PODS. (2002)

2. Yu, C., Popa, L.: Constraint-based XML query rewriting for data integration. In: SIGMOD. (2004)

3. Qin, L., Yu, J.X., Ding, B.: TwigList: make twig pattern matching fast. In: DASFFA. (2007) 